\title{
NOISE ROBUST ADAPTIVE BLIND CHANNEL IDENTIFICATION USING SPECTRAL CONSTRAINTS
}

\author{
Nikolay D. Gaubitch ${ }^{1}$, Md. Kamrul Hasan ${ }^{2}$ and Patrick A. Naylor ${ }^{1}$ \\ ${ }^{1}$ Dept. of Electrical and Electronic Engineering, Imperial College London, UK \\ ${ }^{2}$ Dept. of Electrical and Electronic Engineering, Bangladesh University of Engineering and Technology, Bangladesh \\ E-mail: khasan@eee.buet.ac.bd, \{ndg,p.naylor\}@imperial.ac.uk
}

\begin{abstract}
A class of adaptive blind channel identification algorithms were proposed recently and were demonstrated to be able to successfully identify various types of channels when the observed signals are free from significant levels of measurement noise. In this paper, we provide a study of the effects of noise on these algorithms and show that they misconverge even at moderate values of SNR. We introduce a spectral constraint into the adaptation rule and show that the robustness to noise can be considerably improved. Simulation results are presented for the new algorithm, which demonstrate a significant performance improvement in terms of normalized projection misalignment.
\end{abstract}

\section{INTRODUCTION}

Blind channel identification (BCI) is a commonly occurring problem with several applications in various fields of engineering, in particular where blind de-convolution or source separation is required. Example areas of application include communications where the received signal must be equalized to obtain the transmitted signal [1] and geophysics where the reflectivity of the earth layers is explored by extracting seismic signals from the sensor observations [2]. Our area of interest is in reverberant speech enhancement where the aim is to estimate the acoustic impulse responses blindly from reverberant observations, and then deconvolve to remove the effects of the room $[3,4]$.

Several blind channel identification algorithms have been reported in the literature both for single channel and multichannel environments. For acoustic impulse responses, the latter has been found most attractive due to the added spatial information. A good review of many existing methods can be found in [5]. Recently, a class of adaptive approaches based on the cross-relation between channels was proposed with implementations in the time-domain [6] and in the frequency-domain [7]. Such algorithms are attractive for real-time speech dereverberation applications. However,

This work was supported by the Engineering and Physical Sciences Research Council. the reported methods perform well when very little noise is present $(\mathrm{SNR}>30 \mathrm{~dB}$ ) but the performance degrades as the noise level is increased. In fact, as will be shown here, noise causes these algorithms to misconverge.

In this paper we are concerned with the adaptive blind channel estimation for acoustic impulse responses in the noisy case. In our previous work [8], we have demonstrated that imposing constraints on the adaptive BCI algorithms improves their performance under noisy conditions. Here, we provide an analysis of the effects of noise on the convergence characteristic of the adaptive blind multichannel algorithms. Consequently, we introduce a spectral constraint in the update rule and demonstrate that this can provide significant performance improvement in the presence of noise. This constraint is more general than the one in [8] and can be calculated solely from the channel estimates.

\section{PROBLEM FORMULATION}

Consider a signal, $s(n)$, produced in a noisy and reverberant room, observed by an array of microphones at a distance from the source. The signal received at the $m$ th microphone can be written

$$
\begin{aligned}
& y_{m}(n)=\boldsymbol{h}_{m}^{T} \boldsymbol{s}(n), \\
& x_{m}(n)=y_{m}(n)+\nu_{m}(n),
\end{aligned}
$$

where $\boldsymbol{h}_{m}=\left[\begin{array}{llll}h_{m, 1} & h_{m, 2} & \ldots & h_{m, L}\end{array}\right]^{T}$ is the $L$-tap impulse response of the acoustic path between the source and the $m$ th microphone, $\boldsymbol{s}(n)=[s(n) s(n-1) \ldots s(n-L)]$ and $\nu_{m}$ is ambient noise at the $m$ th microphone. It is assumed that $E\left\{\nu_{l}(n) \nu_{m}(n)\right\}=0$ for $l \neq m$ and $E\left\{\nu_{m}(n) \nu_{m}\left(n-n^{\prime}\right)\right\}=$ 0 for $n^{\prime} \neq 0$. It is also assumed that the noise, $\nu_{m}(n)$, is uncorrelated with the source signal, $s(n)$.

The aim of a blind channel identification algorithm is to form an estimate, $\hat{\boldsymbol{h}}_{m}$, of the impulse responses, $\boldsymbol{h}_{m}$, using only the noisy observations $x_{m}(n), m=1,2, \ldots, M$. This has been shown possible if the following identifiability conditions are satisfied [9]: i) the channels do not share any common zeros and ii) the autocorrelation matrix of the source signal is of full rank. 


\section{ADAPTIVE BLIND CHANNEL IDENTIFICATION}

Here we provide a brief summary of the adaptive BCI algorithms proposed in $[6,7]$ which are based on the cross-relation between the channels [9]

$$
\boldsymbol{y}_{i}^{T}(n) \boldsymbol{h}_{j}=\boldsymbol{y}_{j}^{T}(n) \boldsymbol{h}_{i} . \quad i, j=1,2, \ldots, M
$$

This leads to the following expression:

$$
\boldsymbol{R h}=\mathbf{0},
$$

where $\boldsymbol{h}=\left[\begin{array}{llll}\boldsymbol{h}_{1}^{T} & \boldsymbol{h}_{2}^{T} & \ldots & \boldsymbol{h}_{M}^{T}\end{array}\right]^{T}$ is a vector of the concatenated impulse responses and $\boldsymbol{R}$ is a cross-correlation-like matrix. Thus, the impulse responses can be identified uniquely up to a scaling factor by finding the eigenvector corresponding to the smallest eigenvalue of $\boldsymbol{R}$ [9]. In the presence of noise, an error function can be defined as [6]

$$
e_{i j}(n)=\boldsymbol{x}_{i}^{T} \hat{\boldsymbol{h}}_{j}-\boldsymbol{x}_{j}^{T}(n) \hat{\boldsymbol{h}}_{i} .
$$

The cost function is specified accordingly

$$
J(n)=\sum_{i=1}^{M-1} \sum_{j=i+1}^{M} e_{i j}^{2}(n) .
$$

The estimate of the channels is given by

$$
\hat{\boldsymbol{h}}_{\mathrm{opt}}=\arg \min _{\hat{\boldsymbol{h}}} E\{J(n)\}, \quad \text { subject to }\|\hat{\boldsymbol{h}}\|=1
$$

where $E\{\cdot\}$ denotes expectation. The unit norm constraint shown here is often introduced to avoid the trivial estimate of all zero coefficients. However, it was shown in [10] that the trivial estimate can be avoided if the initial estimation vector is not orthogonal to the true impulse responses. This unconstrained approach is adopted here.

Finally, the channel estimates can be obtained using an adaptive filter for which several efficient algorithms were proposed in [6] and [10]. The simplest approach, which we will use for illustration in this paper is the unconstrained multichannel LMS update equation

$$
\hat{\boldsymbol{h}}(n+1)=\hat{\boldsymbol{h}}(n)-2 \mu \tilde{\boldsymbol{R}}(n) \hat{\boldsymbol{h}}(n),
$$

where $\hat{\boldsymbol{h}}(n)=\left[\hat{\boldsymbol{h}}_{1}^{T}(n) \hat{\boldsymbol{h}}_{2}^{T}(n) \ldots \hat{\boldsymbol{h}}_{M}^{T}(n)\right]^{T}$ is the estimation vector at time $n, \mu$ is a small positive adaptation step-size and $\tilde{\boldsymbol{R}}(n)$ is the instantaneous estimate of $\boldsymbol{R}$ at time $n$. The method and the ideas we present here are general and can be extended to any of the other adaptive filter algorithms, such as the Newton method [6] and the frequency domain algorithms [7].

\section{NOISE EFFECTS IN ADAPTIVE BCI ALGORITHMS}

In this section, an analysis of the noise effect on the blind adaptive channel identification algorithms is presented. To investigate the convergence characteristics of the adaptive BCI algorithms, we rewrite the error function using (2) and (5) as

$$
\begin{aligned}
e_{i j}(n)= & e_{i j}^{y}(n)+e_{i j}^{\nu}(n) \\
= & {\left[\boldsymbol{y}_{i}^{T}(n) \boldsymbol{h}_{j}-\boldsymbol{y}_{j}^{T}(n) \boldsymbol{h}_{i}\right] } \\
& +\left[\boldsymbol{\nu}_{i}^{T}(n) \boldsymbol{h}_{j}-\boldsymbol{\nu}_{j}^{T}(n) \boldsymbol{h}_{i}\right] .
\end{aligned}
$$

Thus, in the presence of noise the error consists of two parts, one due to the signal, $e_{i j}^{y}(n)$ and one due to the noise, $e_{i j}^{\nu}(n)$. We can rewrite the cost function from (6) as

$$
\begin{aligned}
J(n) & =J_{y}(n)+J_{\nu}(n) \\
& =\sum_{i=1}^{M-1} \sum_{j=i+1}^{M}\left(e_{i j}^{y}(n)\right)^{2}+\sum_{i=1}^{M-1} \sum_{j=i+1}^{M}\left(e_{i j}^{\nu}(n)\right)^{2}
\end{aligned}
$$

and consequently, the mean squared error (MSE) estimate of the channel impulse responses can be rewritten

$$
\hat{\boldsymbol{h}}_{\mathrm{opt}}=\arg \min _{\hat{\boldsymbol{h}}} E\left\{J_{y}(n)+J_{\nu}(n)\right\} .
$$

The LMS adaptive algorithm finds the desired solution by moving along the opposite direction of the performance surface according to

$$
\hat{\boldsymbol{h}}(n+1)=\hat{\boldsymbol{h}}(n)-\mu\left(\nabla J_{y}(n)+\nabla J_{\nu}(n)\right),
$$

where $\nabla$ is the gradient operator. The noise-free gradient has been shown to be [6]

$$
\nabla J_{y}(n)=2 \tilde{\boldsymbol{R}}_{y}(n) \hat{\boldsymbol{h}}(n)
$$

and the noise only gradient can be derived in a similar fashion resulting in

$$
\nabla J_{\nu}(n)=2 \tilde{\boldsymbol{R}}_{\nu}(n) \hat{\boldsymbol{h}}(n) .
$$

The mean squared error due to the noise component is given by

$$
\operatorname{MSE}\left(J_{\nu}\right)=\sum_{i=1}^{M} \sum_{j \neq i}^{M} \sigma_{\nu_{i}}^{2} \hat{\boldsymbol{h}}_{i}^{T}(n) \hat{\boldsymbol{h}}_{i}(n) .
$$

Now, the overall gradient, $\nabla J(n)$, needs to approach zero in order for the LMS algorithm to converge, i.e.

$$
\nabla J(n)=\nabla J_{\nu}(n)+\nabla J_{y}(n)=0,
$$

Clearly, the equality in (16) can be satisfied if and only if $\nabla J_{y}(n)=-\nabla J_{\nu}(n)$ because $\nabla J_{\nu}(n) \neq 0$ as seen in (15). From (13) and (14) it can be deduced that $\nabla J_{y}(n) \neq$ $-\nabla J_{\nu}(n)$. Since $\nabla J_{\nu}(n)$ is non-zero in the presence of noise, the adaptive filter is caused to misconverge. An example of this misconvergence is demonstrated in Fig. 3 (a). It was demonstrated in [8] that reducing the step-size only delays the misconvergence. 


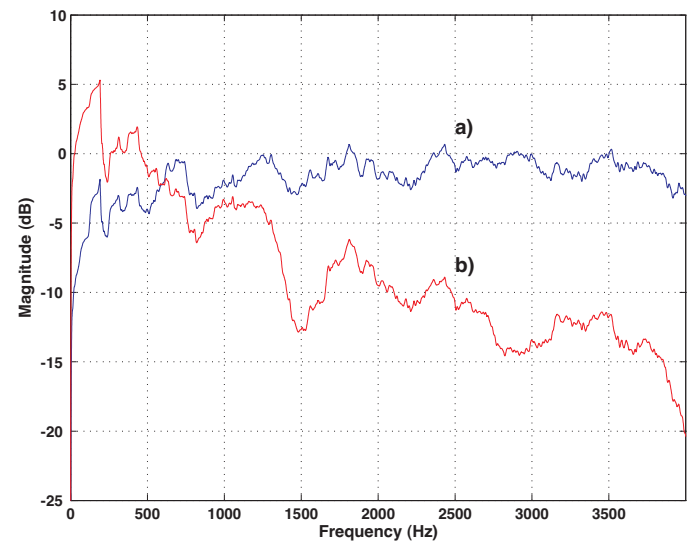

Fig. 1. Smoothed magnitude response of (a) the true channels, $\boldsymbol{h}$, and (b) the misconverged estimates of $\hat{\boldsymbol{h}}$ at $\mathrm{SNR}=20 \mathrm{~dB}$.

\section{SPECTRALLY CONSTRAINED ADAPTIVE BCI}

From our experiments, we note that the misconverged solutions are spectrally weighted versions of the true channels, which show clear low-pass characteristics. An example of this effect is presented in Fig. 1. However, in the case of acoustic impulse responses, it is known, for example from statistical room acoustics [11], that the energy is approximately uniformly distributed over all frequencies when the excitation signal consists of many frequency components [12]. Using this motivation, we now derive a modified adaptation rule by attaching an additional constraint to maintain an approximately equal energy distribution in the smoothed spectrum of the estimated impulse responses in order to improve robustness of the adaptive algorithms in the presence of noise. This constraint, which is calculated directly from the estimated impulse responses, avoids the observed spectral effect by maintaining a uniform spectral energy distribution of the channel estimates.

We propose the following constrained minimization where, for brevity, we have omitted the time index:

$$
\begin{aligned}
\hat{\boldsymbol{h}}_{\mathrm{opt}}= & \arg \min _{\hat{\boldsymbol{h}}} E\{J\} \\
& \text { subject to } \frac{1}{N} \sum_{k=i N}^{i N+N-1}|\hat{H}(k)|^{2}=\mathcal{E}, i=1, \ldots, \mathcal{I}
\end{aligned}
$$

where $\mathcal{I}$ is the number of frames used in the spectral smoothing, $\hat{H}(k)$ is obtained by taking the DFT of $\hat{\boldsymbol{h}}$ and $\mathcal{E}=$ $\frac{1}{M L} \sum_{k=0}^{M L-1}|\hat{H}(k)|^{2}$ is the mean spectral power. The constraint can be written equivalently

$$
\hat{\boldsymbol{h}}^{T} \boldsymbol{\Gamma}_{i} \hat{\boldsymbol{h}}=\mathcal{E},
$$

with

$$
\boldsymbol{\Gamma}_{i}=\boldsymbol{F} \boldsymbol{W}_{i} \boldsymbol{F}^{*},
$$

where $\boldsymbol{F}$ is a DFT matrix and $\boldsymbol{W}_{i}$ is a diagonal matrix with weights for the DFT coefficients and $*$ denotes complex conjugate. Next, we introduce the Lagrange multiplier $\lambda_{i}$ and reformulate the cost function to

$$
J_{C}=J+\sum_{i=0}^{\mathcal{I}} \lambda_{i} e_{\mathrm{p}, i}^{2}
$$

where

$$
e_{\mathrm{p}, i}=\hat{\boldsymbol{h}}^{T} \boldsymbol{\Gamma}_{i} \hat{\boldsymbol{h}}-\mathcal{E}
$$

and $J$ is defined in (10). The overall gradient, $\nabla J_{C}$ is derived, by differentiating with respect to $\hat{\boldsymbol{h}}$, resulting in:

$$
\frac{\partial J_{C}}{\partial \hat{\boldsymbol{h}}}=2 \boldsymbol{R} \hat{\boldsymbol{h}}+4 \sum_{i=0}^{\mathcal{I}-1} \lambda_{i} e_{\mathrm{p}, i} \boldsymbol{\Gamma}_{i} \hat{\boldsymbol{h}} .
$$

Finally, substituting (21) into (12), the update equation for the proposed algorithm is:

$$
\hat{\boldsymbol{h}}(n+1)=\hat{\boldsymbol{h}}(n)-2 \mu\left[\tilde{\boldsymbol{R}}(n) \hat{\boldsymbol{h}}(n)+2 \sum_{i=0}^{\mathcal{I}-1} \lambda_{i} e_{\mathrm{p}, i}(n) \boldsymbol{\Gamma}_{i} \hat{\boldsymbol{h}}(n)\right] .
$$

As can be seen, this contains an additional penalty term in comparison with the original method in (8).

\section{SIMULATIONS AND RESULTS}

We present simulation results to demonstrate the performance of the proposed algorithm in comparison with an existing method. The new approach was implemented both in the time-domain and in the frequency-domain. The results shown here were obtained with the computationally efficient Normalized Multi-Channel Frequency-domain LMS (NMCFLMS) [7]. We used finite acoustic impulse responses generated with the image model [13]. The room dimensions were set to $(6.4 \times 5 \times 4) \mathrm{m}$ with reverberation time $T_{60}=0.5 \mathrm{~s}$ and the impulse responses were truncated to $L=128$ taps. A linear array was assumed with $M=5$ microphones and $0.2 \mathrm{~m}$ spacing between adjacent microphones and $1.5 \mathrm{~m}$ distance from the source to the array. The sampling frequency was $f_{s}=8 \mathrm{kHz}$. The source signal was white Gaussian noise. We used the normalized projection misalignment (NPM) as a performance index, which considers only the misalignment, ignoring the arbitrary scaling effect and is defined as [6]:

$$
\operatorname{NPM}(n)=20 \log _{10}\left(\frac{1}{\|\boldsymbol{h}\|}\left\|\boldsymbol{h}-\frac{\boldsymbol{h}^{T} \hat{\boldsymbol{h}}(n)}{\hat{\boldsymbol{h}}^{T}(n) \hat{\boldsymbol{h}}(n)} \hat{\boldsymbol{h}}(n)\right\|\right) \mathrm{dB} .
$$

Figure 2 shows (a) the true impulse responses, (b) the estimated room impulse responses using the NMCFLMS algorithm and (c) impulse responses estimated with the proposed spectrally constrained algorithm. We have set $\mu=0.8, \lambda_{i}=$ $7.5 \times 10^{-3} \forall i$ which was chosen empirically and the SNR was 


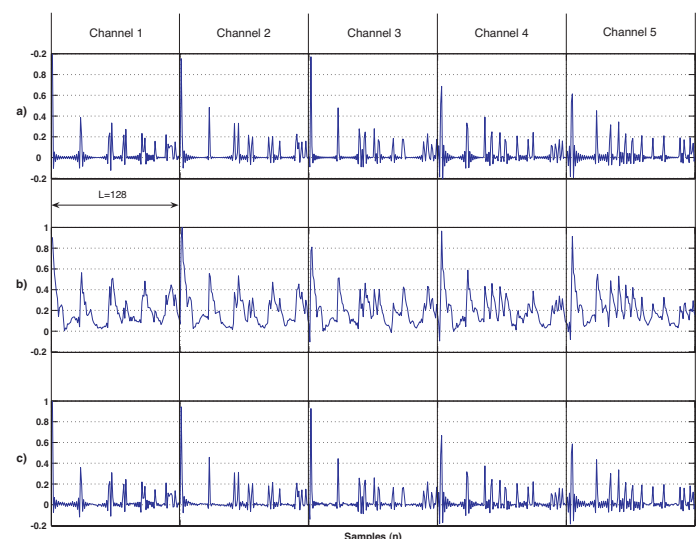

Fig. 2. Impulse responses of the (a) true channels, (b) misconverged estimates using the NMCFLMS and (c) estimates using the Spectrally Constrained NMCFLMS.

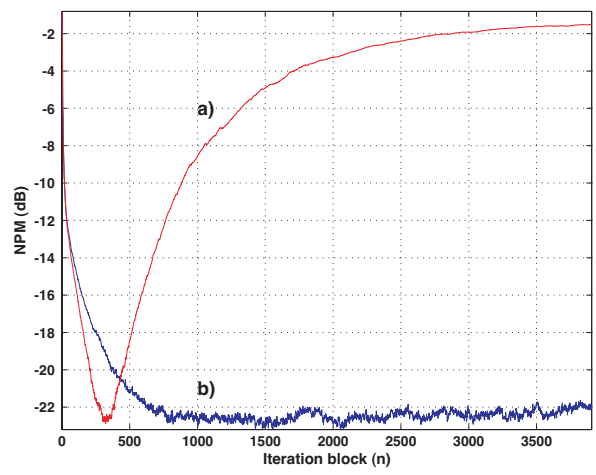

Fig. 3. Normalized projection misalignment for (a) NMCFLMS and (b) Spectrally Constrained NMCFLMS.

$20 \mathrm{~dB}$. As can be seen in Fig. 2 (b), the estimates using the unconstrained adaptive algorithm contain spurious low frequency components. On the contrary, the proposed method has estimated the desired responses accurately. The convergence behavior of the algorithms is shown in Fig. 3 where the NPM is plotted versus the number of iteration blocks for (a) the NMCFLMS and (b) the proposed constrained algorithm. The predicted misconvergence is clearly visible in the NMCFLMS. This misconvergence is also present in other adaptive BCI algorithms as it was demonstrated in [8]. In contrast, it can be seen that the asymptotic convergence performance is improved with the proposed algorithm and is controlled by the noise level.

\section{CONCLUSIONS}

We have investigated the performance of a class of adaptive blind channel identification algorithms for the identification of acoustic impulse responses from noisy observations. We have seen that additive noise causes these algorithms to misconverge and that the reason for this misconvergence is the non-zero gradient of the noise-error surface. We have also demonstrated our observation that the misconverged solutions are spectrally distorted versions of the true impulse responses. Accordingly, a novel approach was proposed for avoiding the misconvergence by attaching a spectral constraint to the adaptation rule. This constraint was based on the assumption of uniform energy distribution over all frequencies. The ability of this approach to avoid misconvergence was demonstrated with experimental results.

\section{REFERENCES}

[1] Z. Xu and M. K. Tsantsanis, "Blind channel estimation for long code multiuser CDMA systems," IEEE Trans. Signal Processing, vol. 48, no. 4, pp. 988-1001, Apr. 2000.

[2] K. F. Kaaresen and T. Taxt, "Image method for efficiently simulating small-room acoustics," Geophysics, vol. 63, no. 6, pp. 2093-2107, Nov. 1998.

[3] S. Subramaniam, A. P. Petropulu, and C. Wendt, "Cepstrumbased deconvolution for speech dereverberation," IEEE Trans. Acoust., Speech, Signal Processing, vol. 4, no. 5, pp. 392-396, Sept. 1996.

[4] S. Gannot and M. Moonen, "Subspace methods for multimicrophone speech dereverberation," EURASIP Journal on Applied Signal Processing, vol. 2003, no. 11, pp. 1074-1090, Oct. 2003.

[5] L. Tong and S. Perreau, "Multichannel blind identification: from subspace to maximum likelihood methods," Proc. IEEE, vol. 86, no. 10, pp. 1951-1968, Oct. 1998.

[6] Y. Huang and J. Benesty, "Adaptive multi-channel least mean square and newton algorithms for blind channel identification," Signal Process., vol. 82, no. 8, pp. 1127-1138, Aug. 2002.

[7] Y. Huang and J. Benesty, "A class of frequency-domain adaptive approaches to blind multichannel identification," IEEE Trans. Signal Processing, vol. 51, no. 1, pp. 11-24, Jan. 2003.

[8] Md. K. Hasan, J. Benesty, P. A. Naylor, and D. B. Ward, "Improving robustness of blind adaptive multichannel identification algorithms using constraints," in Proc. European Signal Processing Conf. (EUSIPCO), Antalya, Turkey, Sept. 2005.

[9] G. Xu, H. Liu, L. Tong, and T. Kailath, "A least-squares approach to blind channel identification," IEEE Trans. Signal Processing, vol. 43, no. 12, pp. 2982-2993, Dec. 1995.

[10] Y. Huang, J. Benesty, and J. Chen, "Optimal step size of the adaptive multichannel $\mathrm{lms}$ algorithm for blind simo identification," IEEE Signal Processing Lett., vol. 12, no. 3, pp. 173176, Mar. 2005.

[11] M. R. Schroeder, "Statistical parameters of the frequency response curves of large rooms," J. Acoust. Soc. Amer., vol. 35, no. 5, pp. 299-303, May 1987.

[12] R. W. Waterhouse, "Statistical properties of reverberant sound fields," J. Acoust. Soc. Amer., vol. 43, no. 6, pp. 1436-1444, May 1968.

[13] J. B. Allen and D. A. Berkley, "Image method for efficiently simulating small-room acoustics," J. Acoust. Soc. Amer., vol. 65, no. 4, pp. 943-950, Apr. 1979. 